\title{
Longitudinal Multisite Randomized Controlled Trial on the Provision of the Acute Stress Syndrome Stabilization Remote for Groups to General Population in Lockdown During the COVID-19 Pandemic
}

\author{
Becker Yael ${ }^{1}$, Estévez María Elena ${ }^{1}$, Pérez María Cristina ${ }^{2}$, Osorio Amalia ${ }^{2}$, Jarero Ignacio $^{1 *}$, and Givaudan \\ Martha $^{1}$
}

${ }^{1}$ Department of Research, Mexican Association for Mental Health Support in Crisis, Mexico

${ }^{2}$ Department of Research, Ágape Desarrollo Integral, Puebla, Mexico

Submission: November 17, 2020; Published: January 18, 2021

*Corresponding author: Ignacio Jarero, Department of Research, Mexican Association for Mental Health Support in Crisis, Mexico City, Mexico

\begin{abstract}
The aim of this longitudinal multisite randomized controlled trial (RCT), using a treatment as usual (TAU) control group design, was to evaluate the effectiveness of the Acute Stress Syndrome Stabilization Remote for Groups (ASSYST-RG) procedure in reducing posttraumatic stress disorder (PTSD), depression, and anxiety symptoms in the general population during the COVID-19 pandemic lockdown in Mexico. A total of 63 adults ( 60 females and 3 males) met the inclusion criteria. Participants' ages ranged from 21 to 73 years old ( $M=43.09$ years). Significant differences between groups were found in all variables. Differences between groups were maintained at follow up assessment. ANOVA for repeated-measures (pre-treatment, post-treatment and follow-up) showed that the ASSYST-RG had a significant effect to reduce Posttraumatic Stress Disorder $\left(\mathrm{F}(2,122)=22.40 \mathrm{p}<.000, \eta_{p}{ }^{2}=.269\right)$. Anxiety and depression showed a significant interaction effect for time and group ( $\mathrm{F}(1,61)$ $\left.=8.89, \mathrm{p}<.00, \eta_{P}{ }^{2}=.127\right)$ and $\left(\mathrm{F}(2,122)=35.04, \mathrm{p}<.001, \eta_{P}{ }^{2}=.365\right)$ respectively. No adverse effects or events were reported by the participants during the procedure administration or at three months follow-up while all participants were still in lockdown. None of the participants showed clinically significant worsening/exacerbation of symptoms after the procedure. This randomized controlled trial provides evidence for the effectiveness, efficacy, feasibility, and safety of the ASSYST-RG in reducing posttraumatic stress, anxiety, and depression symptoms in the general population during the COVID-19 pandemic lockdown.
\end{abstract}

Keywords: COVID-19 general population mental health; Randomized Controlled Trial (RCT); Acute Stress Syndrome Stabilization Remote for Groups; Online group counselling; Online group support; Posttraumatic stress disorder (PTSD); Anxiety; Depression.

\section{Introduction}

On October 26, 2020, the World Health Organization (WHO) reported 42,745,212 confirmed COVID-19 cases with $1,150,961$ deaths around the world and warned that the northern hemisphere was at a critical moment in the face of the COVID-19 pandemic, with a large number of countries registering an exponential growth in infections while Europe overtook South Asia and was placed as the second most affected region in the world with 9,255,953 confirmed cases, only behind America with 19,629,837 confirmed cases, and many European countries (e.g.,
Spain, Germany, France, Italy) were implementing strict measures (e.g., curfews) to contain the increase in COVID-19 cases [1]. The mental health impact of the COVID-19 pandemic related to the governmental and social strategies such as lockdown measures (e.g., stay at home with social isolation, closure of schools, travel restrictions) and increased hygienic practices, especially in Hospitals, around the world are still unknown. Epidemics are associated with a wide range of psychiatric comorbidities like anxiety, panic, depression, and trauma-related disorders 
[2]. Quarantine has been associated with high levels of stress, depression, irritability, and insomnia [3,4]. Two COVID-19 related studies conducted in China with over 60,000 general population' respondents, found rates of $30 \%$ of anxiety, $17 \%$ of depression, and trauma-related distress symptoms, with women and young adults showing significantly higher psychological distress $[5,6]$. In a study conducted with a nationally representative sample of adults from the general population of the Republic of Ireland to determine rates of COVID-19-related posttraumatic stress disorder (PTSD) and the level of comorbidity with depression and anxiety, results showed a rate of $17.7 \%$ of PTSD and a high level of comorbidity with anxiety (49.5\%) and depression (53.8\%) [7]. On a web-based survey of the general population conducted in Italy throughout the internet between March 27th and April 6th, 2020, 18,147 individuals completed the questionnaire. Selected outcomes were post-traumatic stress symptoms (PTSS), depression, anxiety, insomnia, perceived stress, and adjustment disorder symptoms (ADS). Results showed $37 \%$ of the population with PTSS, $17.3 \%$ with depression, $20.8 \%$ with anxiety, $7.3 \%$ with insomnia, $21.8 \%$ with high perceived stress, and $22.9 \%$ with ADS. Being a woman and younger age were associated with all the selected outcomes [8]. In November 2020, the Mental Health Research Canada (MHRC) in partnership with Pollara Strategic Insights (PSI), reported their results of an ongoing longitudinal online survey, conducted with 2,004 Canadian adults. Results showed a significant and alarming increase of anxiety and depression levels due to the COVID-19 outbreak. Their findings also indicate that individuals of the female sex, of younger age, living alone and front-line healthcare workers are more likely to be diagnosed with anxiety and depression [9]. The United Nations (UN) alerted the world about the high risk of a major mental health crisis caused by the COVID-19 pandemic, including but not solely due to the financial difficulties, and recommended the urgent widespread availability and use of mental health care and psychosocial support to address this situation [10]. The use of Telehealth to provide mental health care (TeleMental Health) has had an exponential increase during the COVID-19 pandemic [11]. In 2020 WHO Member States reported the development of stage-specific measures pertaining to the emotional epidemic in order to survey and develop possible measurements to reduce the burden of mental disorders [12].

\section{Adaptive information processing theoretical model}

Briefly stated, the Adaptive Information Processing (AIP) theoretical model is a memory-related model of pathogenesis and change. According to this model, memory networks (patterns of linked memories with similar components) of stored experiences are the basis of both human mental health and human pathology across the clinical spectrum. According to Dr. Francine Shapiro, all human beings have an innate physical information processing system in the brain naturally geared toward health. When the information of an experience (whether positive or negative) is successfully processed, it is adaptively stored in memory networks of experiences that are similar in their components (e.g., sensory input, emotions/feelings, body/somatic sensations). The adaptively stored information forms the adaptive memory networks that support human growth and development via learning. However, disruptions to this processing system due to high arousal states from critical incidents or adverse life experiences result in memories that are inadequately processed and dysfunctionally stored in the brain. These pathogenic memories are stored in isolation and are unable to connect with the adaptive memory networks. The information stored in these neurophysiological memory networks generates the present suffering, difficulties, and symptoms we see in our clients across the clinical spectrum (not only posttraumatic stress disorder). These pathogenic memories can be triggered by similar stimuli or information from the present and the person reacts to the present as if it were the same situation as the past. In other words, the past is present for these persons $[13,14]$. Based in the growing body of research showing that memories can contribute to pathology in many mental disorders, the AIP can be considered a meta-theory and a transdiagnosis model [15].

\section{Syndrome and mental disorder}

In pathology and psychiatry, a syndrome is defined as, "A group of symptoms that together are characteristics of a specific disorder, disease, or the like" [16]. "A mental disorder is a syndrome characterized by a clinically significant disturbance in an individual's cognitive, emotion regulation, or behavior that reflects a dysfunction in the psychological, biological, or developmental process underlying mental functioning. Mental disorders are usually associated with significant distress in social, occupational, or other important activities" (p. 20) [17].

\section{Acute stress disorder and posttraumatic stress disorder}

According to the Diagnostic and Statistical Manual of Mental Disorders ( $5^{\text {th }}$ Edition; DSM-5), Acute Stress Disorder (ASD) and Posttraumatic Stress Disorder (PTSD) are trauma-and stressorrelated disorders in which exposure to a traumatic or stressful event is listed explicitly as a diagnosis criterion [17]. Acute Stress Disorder (ASD) is a mental disorder in which its essential feature, "is the development of characteristic symptoms lasting from 3 days to 1 month following exposure to one or more traumatic events" (p. 281). ASD has five symptom categories 1) Intrusion symptoms, 2) Negative Mood, 3) Dissociative symptoms, 4) Avoidance Symptoms, and 5) Arousal symptoms. To meet the full diagnosis criteria, the client has to show the presence of nine or more symptoms from any of the five categories previously mentioned. It is important to notice that "Approximately half of the individuals who eventually developed PTSD initially present with acute stress disorder" (p. 284) [17].

Posttraumatic Stress Disorder (PTSD) is a mental disorder occurring after exposure to one or more traumatic events (Criterion A) and is characterized by intense reliving of the 
traumatic event through intrusive memories and nightmares (intrusion symptoms; criterion B); avoidance of reminders of the event (avoidance symptoms; criterion $\mathrm{C}$ ); negative alterations in cognitions and mood associated with the traumatic event (criterion D); hypervigilance toward potential threats in the environment (arousal symptoms; criterion E); and in some cases, persistent or recurrent depersonalization symptoms. The Criteria A events included, but are not limited to, threatened or actual physical assault (e.g., childhood physical abuse, physical attack), threatened or actual sexual violence (e.g., forced sexual penetration, alcohol/drug-facilitated sexual penetration, abusive sexual contact, noncontact sexual abuse, sexual trafficking) (p. 274) [17].

Of special interest for this article are the ASD and the PTSD Intrusion Symptoms associated with the traumatic event(s), beginning after the traumatic event occurred, which are 1. Recurrent, involuntary, and intrusive distressing memories of the traumatic event(s). 2. Recurrent distressing dreams in which the content and/or affect of the dream are related to the traumatic event(s). 3. Dissociative reactions (e.g., flashbacks) in which the individual feels or acts as if the traumatic event(s) were recurring. 4. Intense or prolonged psychological distress at exposure to internal or external cues that symbolize or resemble an aspect of the traumatic event(s). 5. Marked physiological reactions to internal or external clues that symbolize or resemble an aspect of the traumatic event(s). "These intrusive memories often include sensory (e.g., sensing the intense heat that was perceived in a house fire), emotional (e.g., experiencing the fear of believing that one was about to be stabbed), or physiological (e.g., experiencing the shortness of breath that one suffered during a near-drowning) components...Some individuals with the disorder do not have intrusive memories of the event itself, but instead experience intense psychological distress or physiological reactivity when they are exposed to triggering events that resemble or symbolize an aspect of the traumatic event" (p. 271, 282-283) [17].

\section{Acute stress syndrome stabilization procedures}

The Acute Stress Syndrome Stabilization (ASSYST) procedures, in both a group and individual formats, are Adaptive Information Processing (AIP)-informed, carefully field-tested, refined, and user-friendly psychophysiological algorithmic approaches, whose references are the EMDR Integrative Group Treatment Protocol for Ongoing Traumatic Stress (EMDR-IGTP-OTS) and the EMDR Protocol for Recent Critical Incidents and Ongoing Traumatic Stress (EMDR-PRECI). These treatment procedures were specifically designed to provide in-person or online support to clients who present Acute Stress Disorder (ASD) or Posttraumatic Stress Disorder (PTSD) intense psychological distress and/ or physiological/somatic reactivity caused by the disorder's intrusion symptoms (e.g., sensory, emotional, or physiological/ somatic components of the intrusive distressing memories) [1623].
The ASSYST procedures give us the possibility of direct, nonintrusive, physiological engagement with the client's distressing/ pathogenic memory and its original eight components (the five sensory inputs, body/somatic sensations, emotions/feelings, and thoughts during the adverse experience). These procedures can be administered within the first hours or days of an event in which the person shows severe symptoms of psychological distress, physiological reactivity, and/or deterioration in current functioning. The objective of these procedures is focused on the client's nervous system activation regulation (stabilization) through the reduction (desensitization) or removal of the activation produced by the sensory, emotional, or physiological (somatic-sensory) components of the intrusive distressing/ pathogenic memories of adverse experiences, to achieve optimal levels of nervous system activation; thus, facilitating the AIP system the subsequent adaptive processing of the information. These procedures follow the Psychological First Aid (PFA) reduction of distress and adaptive functioning objectives and must be administered within a continuum of care context (stepped progression of health care provided in an increasingly intensified manner) to help the person achieve a state of adaptive functioning or to facilitate access to the next level of care when necessary [24]. Due to the COVID-19 mandatory lockdown in Mexico, for this study, we selected the ASSYST-Remote for Groups procedure.

\section{Objective}

The objective of this longitudinal multisite randomized controlled trial (RCT) was to evaluate the effectiveness of the ASSYST-RG in reducing posttraumatic stress disorder, depression, and anxiety symptoms in the general population during the COVID-19 pandemic lockdown in Mexico.

\section{Method}

\section{Study Design}

To measure the effectiveness of the ASSYST-RG on the dependent variables PTSD, Anxiety, and Depression, this study used a two parallel arms longitudinal randomized control trial (RCT) design, comparing ASSYST-RG treatment (T) and treatment as usual (TAU) groups. PTSD, anxiety, and depression symptoms were measured in three-time points for all participants in the study: Time 1. Pre-treatment assessment; Time 2. Post-treatment assessment; and Time 3. Follow-up assessment.

\section{Ethics and Research quality}

The research protocol was reviewed and approved by the EMDR Mexico International Research Ethics Review Board (also known in the United States of America as an Institutional Review Board) in compliance with the International Committee of Medical Journal Editors recommendations, the Guidelines for Good Clinical Practice of the European Medicines Agency (version 1 December 2016) and the Helsinki Declaration of 1975 as revised in 2013. The research quality of this study was based on the Consolidated 
Standards of Reporting Trials (CONSORT) 2010 Statement and the Standard Protocol Items Recommendation for Interventional Trials (SPIRIT) 2013 checklist [25,26].

\section{Participants}

This study was conducted in 2020 in Mexico with the general populating during the COVID-19 pandemic mandatory lockdown. 70 potential participants were recruited. Inclusion criteria were: (a) being adult, (b) being in COVID-19-related total lockdown for at least the last six weeks and experiencing psychological distress and/or physiological/somatic reactivity related to the lockdown, (c) voluntarily participating in the study, (d) not receiving specialized trauma therapy, (e) not receiving drug therapy for posttraumatic stress disorder symptoms. Exclusion criteria were: (a) to have or have had a COVID-19 diagnosis, b) to be living with or taking care of a person(s) with COVID-19, c) ongoing self-harm/ suicidal, or homicidal, or in risk of auto-mutilation, (d) diagnosis of schizophrenia, psychotic or bipolar disorder, (e) diagnosis of dissociative disorder, (f) organic mental disorder, (g) a current, active chemical dependency problem, (h) significant cognitive impairment (e.g., severe intellectual disability, dementia), (i) presence of uncontrolled symptoms due to a medical illness.

\section{Blind randomization, allocation concealment mechanism, and blinding procedure}

Simple randomization using an internet-based system with a 1:1 allocation ratio was used. Two independent assessors blind to treatment conditions conducted the randomization process to avoid allocation influence. One of them provided the random-number list and the other assigned random identification (ID) codes to each random-number in the list to protect the participant's identity. The treatment allocation sequence with the ID codes was concealed using sequentially numbered, opaque, sealed, and stapled envelopes. The safekeeping of the envelopes and the assignment of participants to each arm of the trial was overseen by an independent person not involved in the research study. The treatment allocation of the participants was blinded for the research assistants (all mental health professionals) who conducted the intake interview and the enrollment. All the assessments, from Time 1 to Time 3, were conducted after randomization with the participants answering the selfadministered instruments online and using an identification code instead of their name to protect their identity. An independent assessor received the instruments already answered online and was the safe keeper of all the data.

Seven potential participants were excluded because they were not in a total lockdown. A total of 63 adults ( 60 females and 3 males) met the inclusion criteria. Participants' ages ranged from 21 to 73 years old ( $M=43.09$ years). Participation was voluntary with the participants' signed informed consent in accordance to the Mental Capacity Act 2005. There were 31 participants (30 female and 1 male) in the ASSYST-RG treatment group and 32 participants (30 female and 2 male) in the Treatment as Usual (TAU) group. See Flow Diagram.

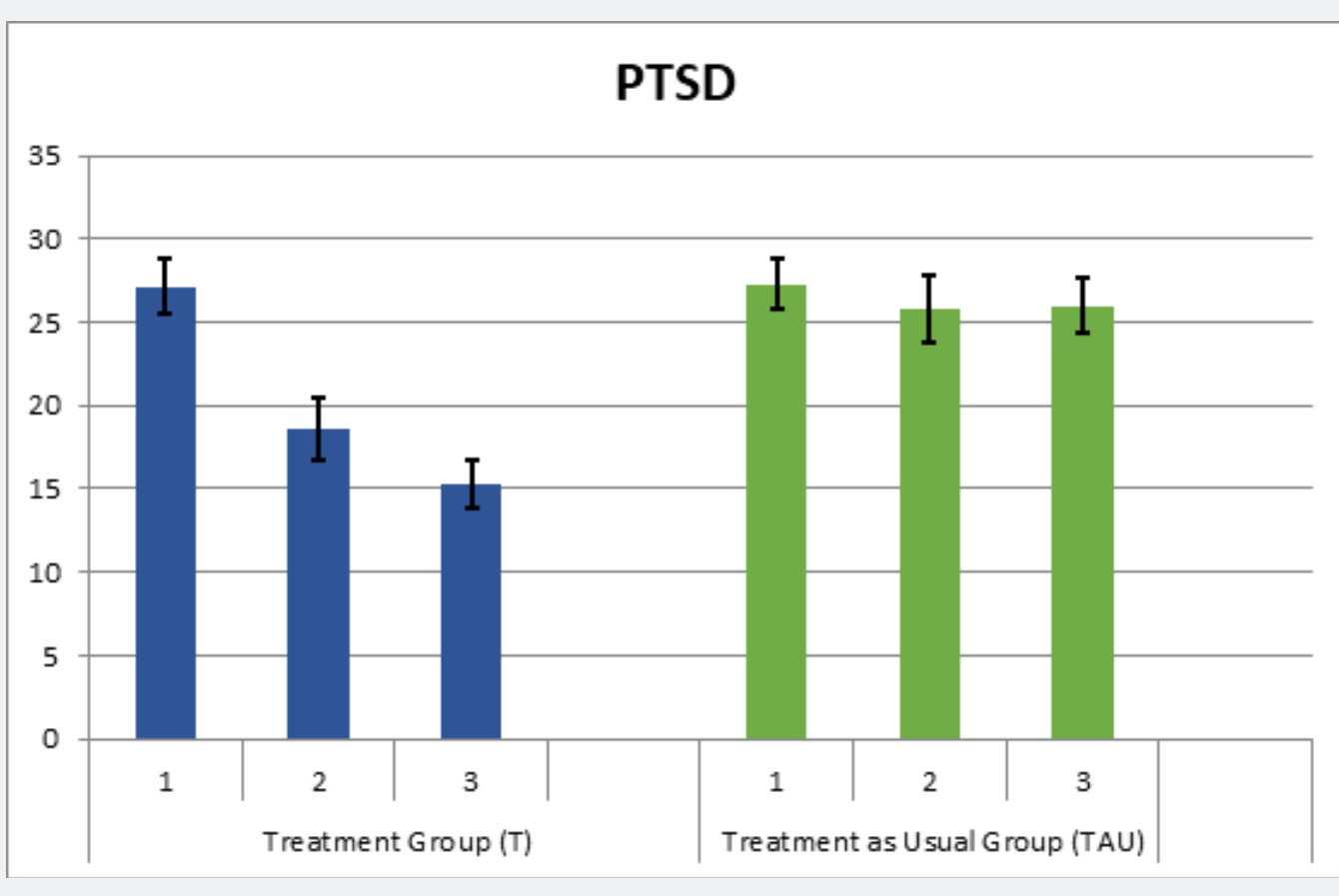

Figure 1: Mean scores and standard error for PTSD symptoms by time and group 


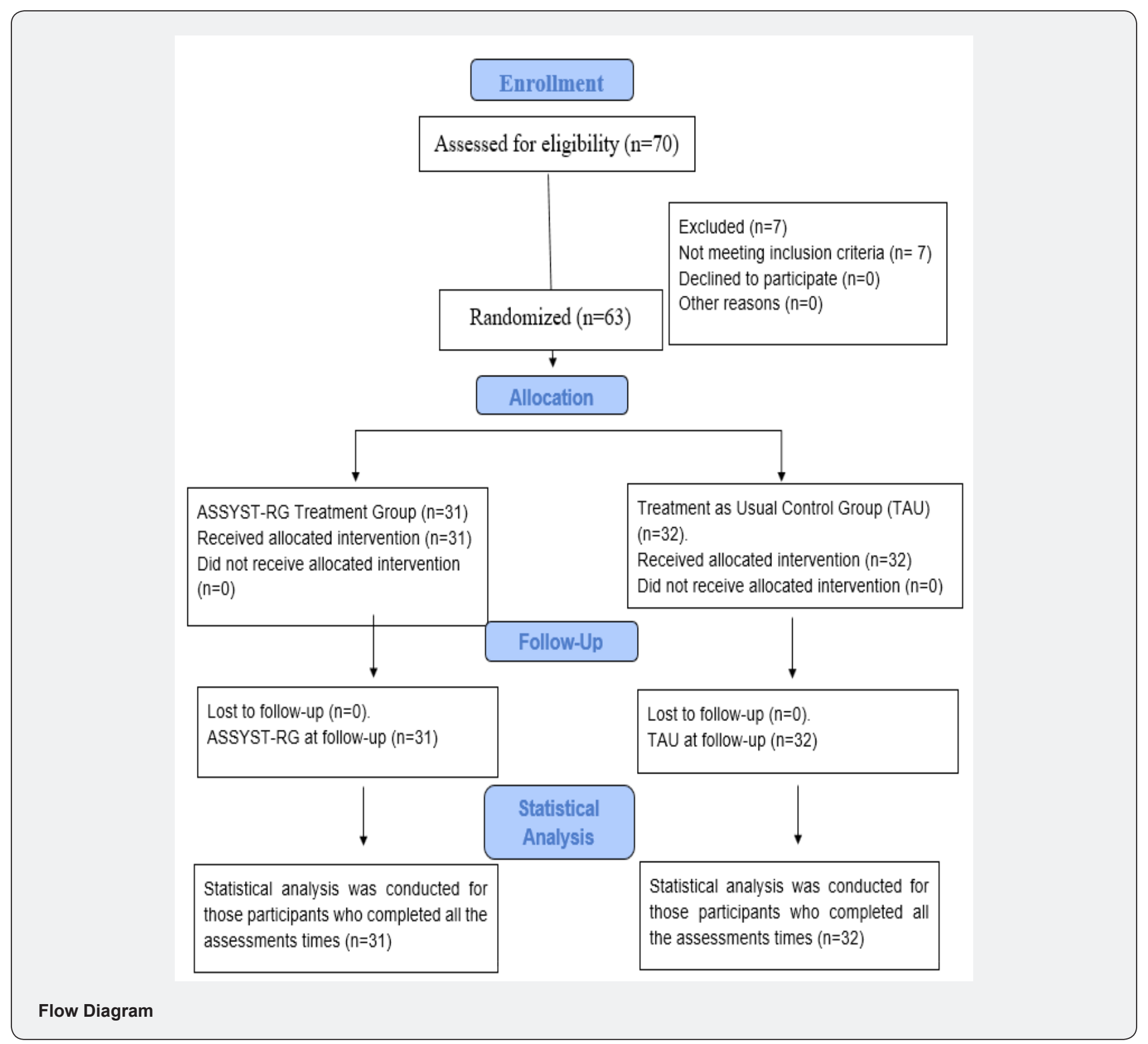

\section{Instruments for psychometric evaluation}

i. We used the Posttraumatic Stress Disorder Checklist for DSM-5 (PCL-5) provided directly by the National Center for PTSD (NCPTSD) and adapted, with the NCPTSD approval, the time interval for symptoms to be the past week instead of the past month $[27,28]$. The instrument was translated and backtranslated to Spanish. It contains 20 items, including three new PTSD symptoms (compared with the PTSD Checklist for DSM-IV) [29]: blame, negative emotions, and reckless or self-destructive behavior. Respondents indicate how much they have been bothered by each PTSD symptoms over the past week (rather than the past month), using a 5 -point scale ranging from $0=$ not at all, $1=$ a little bit, $2=$ =moderately, $3=$ quite a bit, and $4=$ extremely. A total-symptoms score of zero to 80 can be obtained by summing the items. The sum of the scores yields a continuous measure of PTSD symptom severity for symptom clusters and the whole disorder. Psychometrics for the PCL-5, validated against the Clinician-Administered PTSD Scale-5 (CAPS-5) diagnosis, suggest that a score of 31-33 is optimal to determine probable PTSD diagnosis [30,31], and a score of 33 is recommended for use at present. The PCL-5 is intended for a variety of clinical and research assessment tasks, including quantifying PTSD symptom severity, track symptom severity changes longitudinally, measuring the underlying construct of PTSD, establishing a provisional PTSD diagnosis, and estimating the presumed prevalence of PTSD. 
A score decrease between five to ten points demonstrates a clinically significant change. Decision rules for PTSD diagnosis based on PCL-5 are very accurate compared to CAPS diagnosis results. It is important to mention that at the first assessment, before answering the PCL-5, all participants were asked to focus specifically on the worst COVID-19 lockdown-related event that currently bothered them the most; then at each subsequent assessment, they were asked to focus on the same event.

ii. The Hospital Anxiety and Depression Scale (HADS) has been extensively used to evaluate these psychiatric comorbidities in various clinical settings at all levels of healthcare services and with the general population [32,33]. The instrument was translated and back-translated to Spanish. It is a 14-item selfreport scale to measure the anxiety (7 items) and depression (7 items) of patients with both somatic and mental problems using a 4-point Likert scale ranging from 0 to 3. The response descriptors of all items are Yes, definitely (score 3); Yes, sometimes (score 2); No, not much (score 1); No, not at all (score 0). A higher score represents higher levels of anxiety and depression: a domain score of 11 or greater indicates anxiety or depression; 8-10 indicates borderline case; 7 or lower indicates no signs of anxiety or depression.

\section{Procedure}

\section{Enrollment, assessments times, blind data collection, and confidentiality of data}

The invitation to participate in the study was made through social networks (e.g., Facebook and Whatsapp) with a phone number for the intake interview. All participants completed the self-administered instruments online on an individual basis in the three different assessment times. During time 1, research assistants (all mental health professionals) blind to treatment allocation, conducted the intake interview by phone, assessed potential participants for eligibility based on the inclusion/ exclusion criteria, collected their personal data (e.g., name, age, gender, profession, email, telephone), obtained signed informed consent, enrolled participants in the study, sent the participant's data to the data safe keeper independent assessor and sent only the participant's name and email to the treatment allocation envelope's safe keeper. After this procedure and previous randomization, the treatment allocation envelope's safe keeper (not involved in the research study) sent each of the enrolled participants a link to answer the Time 1 (pre- treatment) assessment instruments online, their ID codes, and the treatment dates. The data safe keeper independent assessor received the names, ID codes, and participant's allocation on each arm of the study from the envelope's safe keeper.

Participants were prompted by the data safe keeper independent assessor, using telephone, Whatsapp messages and email to complete the Time 2 and Time 3 questionnaires online. Time 2 post-treatment assessment was conducted online 30 days after the completion of each group treatment with all participants in confinement. Time 3 follow-up assessment was intentionally conducted online 90 days after the two-group treatment' completion (instead of 180 days) to assess participants still being in confinement. All the participant's assessment data was received by the data safe keeper independent assessor, collected, stored, and handled in full compliance with the EMDR Mexico International Research Ethics Review Board requirements to ensure confidentiality. Each study participant gave their consent for access to their data, which was strictly required for study quality control. All procedures for handling, storing, destroying and processing data were in compliance with the Data Protection Act 2018. All persons involved in this research project were subject to professional confidentiality.

\section{Withdrawal from the study}

All research participants had the right to withdraw from the study without justification at any time and with assurances of no prejudicial result. If participants decided to withdraw from the study, they were no longer followed up in the research protocol. There were no withdrawals during this study. See Figure 1 Flow Diagram.

\section{Treatment}

\section{Clinicians and treatment fidelity}

The ASSYST-RG and the TAU were provided in a singlesession per group by eight licensed clinicians (four for each treatment group) formally trained in the ASSYST-RG and the TAU administration in compliance with the APA Guidelines for the Practice of Telepsychology, using a HIPAA-compliant platform. Videos and screenshots of each session were allowed and accepted by each participant in the informed consent and were used by independent raters to monitor both treatment fidelity and adherence to all steps of the procedures.

\section{Treatment description and safety measure}

The intake interview was made by phone for each potential group participant. The ASSYST-RG treatment participants received an average of 60 minutes of online treatment, provided during a single online group session. The treatment as usual (TAU) participants received an average of 60 minutes of online psychoeducation about the possible confinement symptoms, stress management, and self-care techniques, provided during a single online group session. The ASSYST-RG treatment focused only on the distressing memories related to their experience of lockdown due to COVID-19 and did not address any other memories. During this process, participants followed directions from the team leader and worked quietly and independently on their distressing memories. The ASSYST-RG team leader asked each of the participants to, "Run a mental movie of everything that's happened during the pandemic lockdown, from right before the beginning until today, or even looking into the future." The treatment's first target was the worst part of the mental movie, 
subsequently, the team leader asked participants to run the mental movie again and target any other memory that was currently distressing, noticing associated emotions/feelings and body sensations. Participants in this study used the Butterfly Hug (BH) ten times on average as a self-administered bilateral stimulation method to process the intrusive distressing memories. Most participants processed more than one distressing memory [34].

As a safety measure, participants were instructed to immediately report to the clinician's coordinator any adverse effects (e.g., symptoms of dissociation, fear, panic, freeze, shut down, collapse, fainting), events (e.g., suicide ideation, suicide attempts, self-harm, homicidal ideation), or symptoms worsening, during the entire study time-frame.

\section{Examples of worst experiences reprocessed during the online treatment}

Losing the meaning of life and feeling unmotivated. Feeling completely anxious and anguished. Missing what life was like before all this and feeling like it will never end. Feeling trapped and like I could not breathe, not hearing a sound on the streets. The fear of everything or in general (losing my job, a family member dying, getting sick, dying myself, spending all my money, and not having anything to eat). Losing close loved ones because of the virus. Contracting the virus and having to spend time isolated in the hospital. Dying alone in the hospital.

\section{Statistical analysis}

Analysis of variance (ANOVA) for repeated measurements (pre-TIME 1, post-TIME 2 and follow up-TIME 3) with two groups, treatment ( $\mathrm{T}$ ) and treatment as usual (TAU), was applied to analyze the effects of the treatment on PTSD, Anxiety, and Depression; Within and between means comparisons using t-test plus Cohen's $\mathrm{d}$ were also included reporting the effect size.

\section{PTSD}

ANOVA for repeated measures of the Posttraumatic Stress Disorder Checklist for DSM-5 (PCL-5) showed a significant effect for time $\left(\mathrm{F}(2,122)=22.40 \mathrm{p}<.000, \eta_{P}{ }^{2}=.269\right)$, a significant effect for group $\left(\mathrm{F}\left(1,61=8.42, \mathrm{p}<.005, \eta_{p}{ }^{2}=.121\right)\right.$ and a significant interaction between time and group, $(\mathrm{F}(2,122)=13.79, \mathrm{p}<.001$, $\left.\eta_{p}{ }^{2}=.184\right)$. Between means comparison did not show significant differences for time 1. For time 2 significant differences between the Treatment Group (T) and the Treatment as usual (TAU) group were found, $\mathrm{t}(61)=-2.65, \mathrm{p}<.01, \mathrm{~d}=.67$. Significant differences among groups continued over the time, $\mathrm{t}(61)=-4.94 \mathrm{p}<.001, \mathrm{~d}$ $=1.24$ with a large effect size. In the Treatment group $(\mathrm{T})$ mean scores showed a significant decrease after the treatment (time 2), $\mathrm{t}(30)=5.29, \mathrm{p}<.001, \mathrm{~d}=.612$ and between the Post-treatment (time 2) and the Follow-up (time 3), t (30)=2.79, $\mathrm{p}<.01, \mathrm{~d}=.24$. For the Treatment as usual (TAU) group no significant differences were found among the possible comparisons. See Table 1 and Figure 1.

Table 1: Mean scores (M) and standard deviations (SD) for Treatment Group (T) and Treatment as usual group (TAU), on Pre-treatment, Post-treatment, and Follow-up assessments.

\begin{tabular}{|c|c|c|c|c|c|c|}
\hline & \multicolumn{2}{|c|}{ Time 1 } & \multicolumn{2}{c|}{ Time 2 } & \multicolumn{2}{c|}{ Time 3 } \\
\hline & M & SD & M & SD & M & SD \\
\hline PTSD & & & & & & \\
\hline $\mathrm{T}$ & 27.16 & 9.48 & 18.54 & 10.42 & 15.25 & 8.14 \\
\hline TAU & 27.28 & 8.33 & 25.78 & 11.17 & 26.00 & 9.06 \\
\hline Anxiety & & & & & & \\
\hline $\mathrm{T}$ & 10.77 & 4.52 & 8.51 & 4.17 & 8.32 & 3.20 \\
\hline TAU & 10.56 & 2.77 & 11.15 & 2.59 & 12.03 & 2.00 \\
\hline Depression & & & & & & \\
\hline $\mathrm{T}$ & 5.58 & 3.22 & 3.83 & 2.87 & 3.67 & 2.31 \\
\hline TAU & 5.50 & 2.40 & 7.37 & 3.26 & 8.53 & 2.73 \\
\hline
\end{tabular}

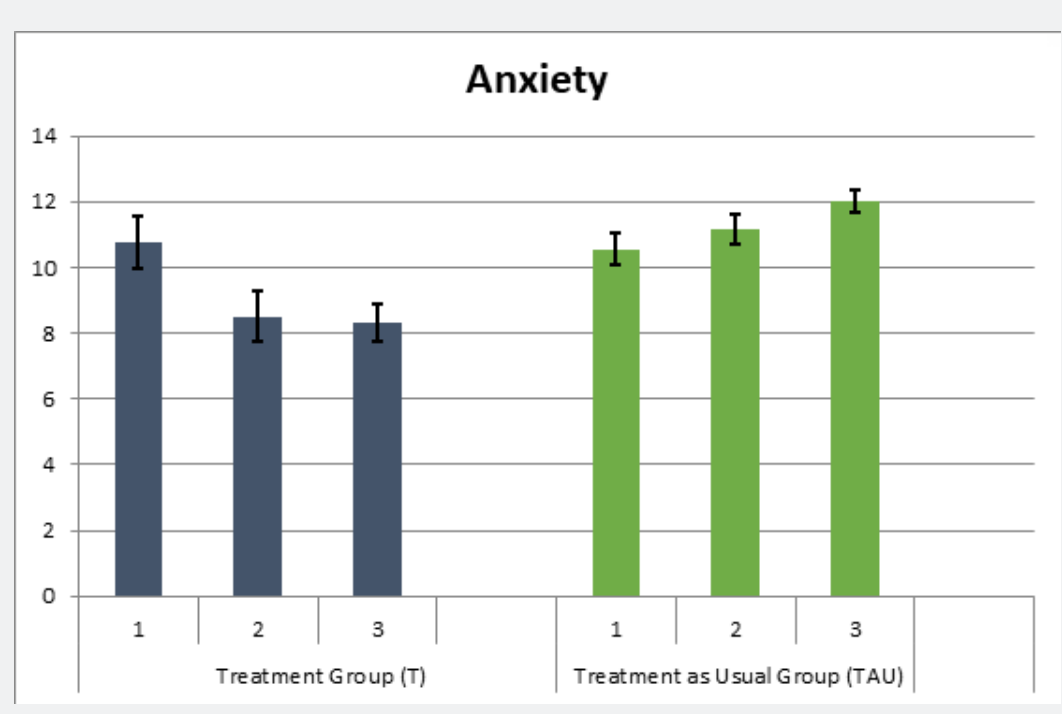

Figure 2: Mean scores and standard error for Anxiety by time and group. 


\section{Psychology and Behavioral Science International Journal}

\section{Anxiety}

Repeated measures analysis of variance (ANOVA) for anxiety did not show a significant effect for time. Significant effect for group was found $\left(\mathrm{F}(1,61)=8.89, \mathrm{p}<.00, \eta_{P}^{2}=.127\right)$ as well as significant interaction effect for time and group $(F(2,122)=11.94$ $\mathrm{p}<.001, \eta_{P}^{2}=.164$. Mean comparison between groups did not show significant differences at Pre-treatment (time 1). For Post treatment (time 2) significant differences between groups were also found, $\mathrm{t}(61)=-3.02, \mathrm{p}<.01, d=.76$, differences between Posttreatment (time 2) and Follow-up (time 3) were also significant (72) $=-5.51, \mathrm{p}<.001, d=.1 .39$. Within comparisons showed that for the Treatment group ( $\mathrm{T}$ ) there was significant differences comparing Pre-treatment (time 1) and Post-treatment (time 2), $\mathrm{t}(30)=2.76, \mathrm{p}<.01, d=.36$. No differences were found between Post-treatment (time 2) and Follow-up (time 3) assessments in this group. For the TAU group significant differences were found between the Post-treatment (time 2) and the Follow-up (time 3) measurements, t (31) $=-2.7, \mathrm{p}<.001, d=. .26)$. See Table 1 and
Figure 2 .

\section{Depression}

No significant effect was found for time through ANOVA repeated measurement analysis. Results showed significant interaction effects between time and group $(F(2,122)=35.04$, $\left.\mathrm{p}<.001, \eta_{p}{ }^{2}=.365\right)$ and significant effects for group, $\mathrm{F}(1,61)=$ 20.02, $\mathrm{p}<.001, \eta_{P}{ }^{2}=.247$. Comparison between groups did not showed significant differences at Pre-treatment (time 1). At Posttreatment and at Follow-up assessments, significant differences were found between groups, $\mathrm{t}(61)=-3.02, \mathrm{p}<.001, d=1.15$ and $\mathrm{t}(61)=-7.59, \mathrm{p}<.001, d=1.91$, respectively. For the Treatment group (T) significant differences were found between Pretreatment (time 1) and Post-treatment (time 2), t (30) = 3.93, $\mathrm{p}<.001, d=.40$. For the TAU group, significant differences were found between Pre-treatment (time 1) and Post-treatment (time 2) as well as between Post-treatment (time 2) and Follow-up (time3) assessments, $\mathrm{t}(31)=-3.42, \mathrm{p}<.01, d=.46$ and $\mathrm{t} \mathrm{(31)} \mathrm{=} \mathrm{-}$ $4.28, \mathrm{p}<.001, d=.27$ respectively. See Table 1 and figure 3.

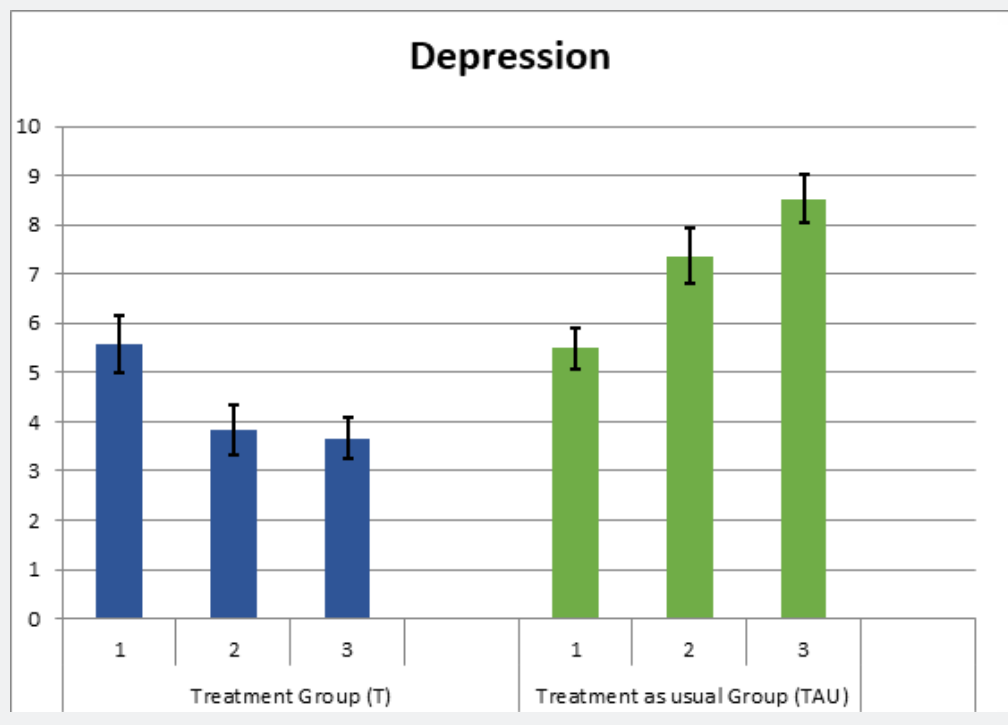

Figure 3: Mean scores and standard error for Depression by time and group.

\section{Safety and symptoms worsening}

There were no adverse effects (e.g., symptoms of dissociation, fear, panic, freeze, shut down, collapse, fainting), or events (e.g., suicide, suicide attempts, self-harm, homicidal ideation) reported by the participants during treatment or at three months post-treatment follow-up while all participants were still in COVID-19 lockdown. None of the participants showed worsening/ exacerbation of symptoms on the PCL-5 or HADS scores during the study frame.

\section{Discussion}

The aim of this longitudinal multisite randomized controlled trial (RCT), using a treatment as usual (TAU) control group design, was to evaluate the effectiveness of the Acute Stress Syndrome Stabilization Remote for Groups (ASSYST-RG) procedure in reducing posttraumatic stress disorder (PTSD), depression, and anxiety symptoms in the general population during the COVID-19 pandemic lockdown in Mexico. A total of 63 adults (60 females and 3 males) met the inclusion criteria. Participants' ages ranged from 21 to 73 years old ( $M=43.09$ years). Participation was voluntary with the participants' signed informed consent in accordance to the Mental Capacity Act 2005. In this study, the two treatments were provided by eight licensed clinicians (four for each treatment condition) formally trained in the ASSYST-RG and the TAU procedures, in compliance with the APA Guidelines for the Practice of Telepsychology, using a HIPAA-compliant platform. 
Pre-treatment measurements for PTSD, anxiety and depression showed similar scores for both groups. After the Acute Stress Syndrome Stabilization Remote for Groups (ASSYST-RG) procedure, significant differences between groups were found in all variables. Significant differences between groups were maintained at follow up assessment. Results for posttraumatic stress disorder (PTSD) showed a continuous decrease over time in the treatment group while in the treatment as usual (TAU) control group scores remain in the same range without significant changes. Anxiety results showed significant effects attributed to the Acute Stress Syndrome Stabilization Remote for Groups (ASSYST-RG) procedure. The size of the effect of the differences between groups increased over the time. Observing the results in the Treatment group there is a significant decrease of anxiety after the treatment and the level of anxiety did not increase in the follow up. It is interesting to note that for the group that received treatment as usual (TAU) there was a significant difference between the second and the follow up measurement due to an increase in the mean. In the case of Depression, a similar pattern was observed in the group that received the procedure significant decrease was observed after the treatment and in the follow up the level of depression remains similar to the post-treatment. In the group that received treatment as usual, the scores for depression increased significantly over the time.

Figures showed clearly a similar pattern for PTSD and Anxiety; significant decrease after treatment that is maintained in the follow up, with no changes in PTSD, once it decreased, and a slight but significant increase of anxiety at follow up for the group that received treatment as usual. In the case of depression, there was an expected effect of the Acute Stress Syndrome Stabilization Remote for Groups (ASSYST-RG) procedure, however in the group that received the treatment as usual, as a control group, the scores of depression gradually increased, probably due to the losses and grief associated with the pandemic. Lockdown is viewed around the world as an effective strategy to slow the spread of the COVID virus but this measure takes a big toll on the populations as it creates fear, anxiety and uncertainty, also the economic recession that derives from this creates financial pressure on families leading to violence and abuse [35]. In Mexico the population became more prone to mental disorders due to restriction of freedom of movement.

Telehealth to provide home-based mental health care has expanded exponentially during the COVID-19 pandemic and generally satisfies patient's care expectations [36] It is also a powerful tool to generate a rapid response to those in need. A growing body of research with war veterans in the United States has examined the use of telemental health [37]. However, high-quality research on video Telehealth services to provide psychiatric, psychological counseling, or evidencebased psychotherapies to treat mental disorders, mental health conditions, or prolonged adverse experiences (e.g., ongoing or prolonged traumatic stress) in the general population is still in its infancy in comparison with the in-person existing research. The one-to-one, in-office traditional mental care is not enough to meet the actual mental health needs of the worldwide population. Therefore, there is an urgent and ethical need for evidence-based, time-limited, culturally sensitive, and costeffective online approaches for the resolution of memories of adverse experiences producing the pathogenic symptoms, that can safely and effectively be delivered cross-culturally in group and individual formats to upscale the TeleMental Health options. To reduce cultural resistance and increase acceptance, those approaches must be minimally intrusive, not requiring a detailed narrative of the traumatic experience, verbal or written disclosure of details, the prolonged reliving of traumatic experiences, or homework relieving the adverse experience [38]. Even though TeleMental Health is helping many in distress during Covid-19, there are still barriers to address because there are places in the world, especially in developing countries, where, due to low socioeconomic status or lack of internet infrastructure 3.6 billion people still remain offline and are unable to access such help [39]. It is also very important to make clear that the majority of the world's population lacks the appropriate orientation to handle the emotional crisis they are facing due to the situation caused by COVID-19, and so, there is a need to reconceptualize and educate on what positive and negative emotions refer to [40].

\section{Conclusion, Limitations, and Future Directions}

During the COVID-19 pandemic and mandatory lockdown measures, studies have shown high rates of posttraumatic stress disorder, posttraumatic stress symptoms, anxiety, depression, insomnia, perceived stress, and adjustment disorder symptoms, independently from previous mental illness or childhood trauma. Therefore, the need for high-quality research on evidencebased time-limited, culturally sensitive, and cost-effective online approaches to address this world-wide major mental health crisis is crucial. The study results showed that the ASSYST-RG can effectively and safely be provided online in a group format to the adult general population living in a pandemic lockdown to reduce PTSD, anxiety, and depression symptoms. It also shows that technology used mostly for leisure can now be effectively used as a tool to educate, work, maintain relationships and support and reduce anguish during a crisis at a distance. We believe that this RCT may also contribute to academia and research regarding the future for this and other EMDR protocols with the same purpose. Limitations of this study are the small sample and the 90-days follow-up (that was intentionally done to assess participants while they were still in confinement). We recommend more randomized controlled trials with larger samples and with follow-up at six or twelve months to evaluate the long-term treatment effects.

\section{References}

1. World Health Organization (2020) Coronavirus disease (COVID-19) Situation Report 115.

2. Tucci V, Moukaddam N, Meadows J, Shah S, Galwanker SC, et al. (2017) 
The forgotten plague: Psychiatric manifestations of ebola, zika, and emerging infectious diseases. J Global Infect Dis 9: 151-156.

3. DiGiovanni C, Conley J, Chiu D, Zaborski J (2004) Factors influencing compliance with quarantine in Toronto during the 2003 SARS outbreak. Biosecur Bioterror 2(4): 265-272.

4. Hawryluck L, Gold WL, Robinson S, Pogorski S, Galea S, et al. (2004) SARS control and psychological effects of quarantine, Toronto, Canada. Emerg Infect Dis 10(7): 1206-1212.

5. Wang C, Pan R, Wan X, Tan Y, Xu L, et al. (2020) Immediate Psychological Responses and Associated Factors during the Initial Stage of the 2019 Coronavirus Disease (COVID-19) Epidemic among the General Population in China. Int J Env Res Public Heal 17(5): 1729.

6. Qiu J, Shen B, Zhao M, Wang Z, Xie B, et al. (2020) A nationwide survey of psychological distress among Chinese people in the COVID-19 epidemic: Implications and policy recommendations. Gen Psychiatry 33(2): 19- 21

7. Kratzias T, Shevlin M, Murphy J, McBride O, Ben-Ezra M, et al. (2020) Posttraumatic Stress Symptoms and Associated Comorbidity During the COVID-19 Pandemic in Ireland: A Population Based Study. Journal of Traumatic Stress 33: 365-370.

8. Rossi R, Socci V, Talevi D, Mensi S, Niolu C, et al. (2020) COVID-19 Pandemic and Lockdown Measures Impact on Mental Health Among the General Population in Italy. Front Psychiatry 11: 790.

9. Mental Health Research Canada (2020) National poll on impact of COVID-19: Three studies in our year-long series. Poll report \#3.

10. Policy Brief: COVID-19 and the Need for Action on Mental Health (2020) United Nations.

11. Liu S, Yang L, Zhang C (2020) Online mental health services in China during the COVID-19 outbreak. Lancet Psychiatry 7: E17-E18.

12. Ransing R, Adiukwu F, Pereira-Sanchez V, Ramalho R, Orsolini L, et al. (2020) Early career psychiatrists' perspectives on the mental health impact and care of the COVID-19 pandemic across the world. Asian Journal of Psychiatry Volume 51: 102085.

13. Shapiro F (2018) Eye movements desensitization and reprocessing Basic principles, protocols, and procedures ( $3^{\text {rd }}$ Edtn.) Guilford Press, United States.

14. Centonze D, Siracusane A, Calabresi P, Bernardi G (2005) Removing pathogenic memories: a neurobiology of psychotherapy. Mol Neurobiol 32: $123-132$.

15. Hase M, Balmaceda UM, Ostacoli L, Liebermann P, Hofmann A (2017) The AIP Model of EMDR Therapy and Pathogenic Memories. Front Psychol 8: 1578.

16. Definition For syndrome. Dictionary.com.

17. American Psychiatric Association (2013) Diagnostic and statistical manual of mental disorders ( $5^{\text {th }}$ Edtn.) Arlington, VA, United States.

18. Jarero I, Artigas L (2018) AIP model-based Acute Trauma and Ongoing Traumatic Stress Theoretical Conceptualization (2 ${ }^{\text {nd }}$ Edtn). Iberoamerican Journal of Psychotraumatology and Dissociation 10(1): $1-10$.

19. Jarero I, Schnaider S, Givaudan M (2019) EMDR Protocol for Recent Critical Incidents and Ongoing Traumatic Stress with First Responders: A Randomized Controlled Trial. Journal of EMDR Practice and Research 13(2):100-110.

20. Jiménez G, Becker Y, Varela C, García P, Nuño MA, et al. (2020) Multicenter Randomized Controlled Trial on the Provision of the EMDR-PRECI to Female Minors Victims of Sexual and/or Physical Violence and Related PTSD Diagnosis. American Journal of Applied Psychology 9(2): 42-51.
21. Jarero I, Givaudan M, Osorio A (2018) Randomized Controlled Trial on the Provision of the EMDR Integrative Group Treatment Protocol Adapted for Ongoing Traumatic Stress to Patients with Cancer. Journal of EMDR Practice and Research 12(3): 94-104.

22. Osorio A, Pérez MC, Tirado SG, Jarero I, Givaudan M (2018) Randomized Controlled Trial on the EMDR Integrative Group Treatment Protocol for Ongoing Traumatic Stress with Adolescents and Young Adults Patients with Cancer. American Journal of Applied Psychology 7(4): 50-56.

23. Jarero I, Uribe S, Artigas L, Givaudan M (2015) EMDR protocol for recent critical incidents: A randomized controlled trial in a technological disaster context. Journal of EMDR Practice and Research 9(4): 166.

24. Everly G, Lating JM (2017) The Johns Hopkins Guide to Psychological First Aid. Johns Hopkins University Press, United States.

25. Consolidated Standards of Reporting Trials (CONSORT) 2010 Statement. BMJ 340: c869

26. Standard Protocol Items Recommendation for Interventional Trials (SPIRIT) 2013 Checklist.

27. Weathers FW, Litz BT, Keane TM, Palmieri PA, Marx BP, et al. (2013) The PTSD Checklist for DSM-5 (PCL-5) Scale available from the National Center for PTSD.

28. Bovin MJ, Marx BP, Weathers FW, Gallagher MW, Rodriguez P, et al. (2016) Psychometric properties of the PTSD checklist for diagnostic and statistical manual of mental disorders- Firth edition (PCL-5) in veterans. Psychol Assess 28(11): 1379-1391.

29. American Psychiatric Association (2000) Diagnostic and statistical manual of mental disorders (4th ed. Text Revised) Arlington, VA, United States.

30. Weathers FW, Blake DD, Schnurr PP, Kaloupek DG, Marx BP, et al. (2013a) Clinician-administered PTSD Scale for DSM-5. National Center for Posttraumatic Stress Disorder, Boston, United States.

31. Franklin CL, Raines AM, Cucurullo LA, Chambliss JL, Maieritsch KP, et al. (2018) 27 ways to meet PTSD: Using the PTSD-checklist for DSM-5 to examine PTSD core criteria. Psychiatry Research 261: 504-507.

32.Zigmond AS, Snaith RP (1983) The Hospital Anxiety and Depression Scale. Acta Psychiatrica Scandinavica 67: 361-370.

33. Ying Lin C, Pakpour AH (2017) Using Hospital Anxiety and Depression Scale (HADS) on patients with epilepsy: Confirmatory factor analysis and Rasch models. Seizure (45): 42-46.

34. Artigas L, Jarero I (2014) The Butterfly Hug. In: M Luber (Ed.) Implementing EMDR Early Mental Health Interventions for Man-Made and Natural Disasters Springer, New York, United States pp.127-130.

35. Poudel K, Subedi P (2020) Impact of COVID-19 pandemic on socioeconomic and mental health aspects in Nepal. International Journal of Social Psychiatry 66(8): 748-755.

36. Kruse CS, Krowski N, Rodriguez B (2017) Telehealth and patient satisfaction: A systematic review and narrative analysis. BMJ Open 7: e016242-e016242.

37. Rosen CS, Morland LA, Glassman LH, Marx BP, Weaver K, et al. (2020) Virtual Mental Health Care in the Veterans Health Administration's Immediate Response to Coronavirus Disease-19. American Psychologist. Advance online publication.

38. Pérez MC, Estévez ME, Becker Y, Osorio A, Jarero I, et al. (2020) Multisite Randomized Controlled Trial on the Provision of the EMDR Integrative Group Treatment Protocol for Ongoing Traumatic Stress Remote to Healthcare Professionals Working in Hospitals During the COVID-19 Pandemic. Psychology and Behavioral Science International Journal 15(4): 1-12. 
39. Zhai Y (2020) A call for addressing barriers to telemedicine: health disparities during the COVID-19 pandemic. Psychotherapy and Psychosomatics, 1.
40. Goleman D, Bennett L, Barlow Z (2012) Ecoliterate: How educators are cultivating emotional, social, and ecological intelligence. John Wiley \& Sons.

\section{Your next submission with Juniper Publishers will reach you the below assets}

- Quality Editorial service

- Swift Peer Review

- Reprints availability

- E-prints Service

- Manuscript Podcast for convenient understanding

- Global attainment for your research

- Manuscript accessibility in different formats

( Pdf, E-pub, Full Text, Audio)

- Unceasing customer service

Track the below URL for one-step submission https://juniperpublishers.com/online-submission.php 